\title{
Curie Transition in Poled and Unpoled Copolymer of Vinylidene Fluoride and Tetrafluoroethylene
}

\author{
Yukinobu MURATA \\ Osaka Prefectural Technical College, 26-12, Saiwai-cho, \\ Neyagawa, Osaka 572, Japan
}

(Received August 20, 1986)

\begin{abstract}
Copolymers of vinylidene fluoride (VDF) and tetrafluoroethylene (TeFE) with TeFE content ranging from 17.8 to $30.7 \mathrm{~mol} \%$ were studied by thermal, dynamic mechanical, and thermally stimulated current measurements. Below the melting point, a small endotherm was observed in differential thermal analysis. Corresponding to the endotherm, a crystalline relaxation, designated as $T_{t}$, was observed by mechanical measurements. It was inferred that the endotherm and the $T_{\mathrm{t}}$ mechanical relaxation were due to the Curie transition. The Curie points of these copolymers were about $50 \mathrm{~K}$ lower than those of VDF-trifluoroethylene (TrFE) copolymers with the same VDF content. In X-ray measurements, it was confirmed that these copolymers took the all-trans crystal structure similar to that of PVDF form I of which the crystal lattice expanded in $a$ and $b$-axis directions and was disordered in the $c$-axis repeat in comparison with that for VDFTrFE copolymers. The Curie temperature for poled samples increased by about $50 \mathrm{~K}$. The poling procedure made the crystal lattice compact in $a$ - and $b$-axis directions and the $c$-axis repeat regular; this seems to be related to the considerable increase in the Curie temperature.

KEY WORDS Curie Transition / Vinylidene Fluoride-Tetrafluoroethylene

Copolymer / Endotherms / Mechanical Relaxation / Poling Procedure /

Crystal Structure / Order /
\end{abstract}

Numerous studies have been made on poly(vinylidene fluoride) (PVDF) and its copolymers with trifluoroethylene (TrFE) for their ferroelectric properties. It has been confirmed that the origin of ferroelectricity of PVDF and VDF-TrFE copolymer is due to the polar crystal of form $\mathrm{I}^{1,2}$ and similar structures, ${ }^{3,4}$ respectively, where the molecular chain takes the all-trans conformation. A copolymer of VDF and tetrafluoroethylene (TeFE) crystallizes into the crystal $^{5,6}$ similar to form I and shows the ferroelectric properties. $^{7,8}$ The Curie temperature is an important character of ferroelectric material, being found for VDF-TrFE copolymers by $\mathrm{X}$-ray, ${ }^{3,4}$ dielectric, ${ }^{9,12}$ dynamic mechanical, ${ }^{10,11}$ and thermal measurements. ${ }^{10,12} \mathrm{Re}$ cently, Lovinger observed the Curie transition for the VDF-TeFE copolymer with 19 $\mathrm{mol} \%$ by X-ray measurements ${ }^{13}$ and discussed the thermal and dielectric behavior. ${ }^{14}$ In the preliminary study, ${ }^{15}$ we found the Curie transitions for these copolymers with varied TeFE content by thermal and mechanical measurements. However, in our dielectric study $^{16}$ of these copolymers, no Curie transition was observed. As the temperature increased, the values of $\varepsilon^{\prime}$ and $\varepsilon^{\prime \prime}$ increased and showed peaks near melting points. This dielectric behavior was attributed to the space charge polarization due to the ionic impurity. On the other hand, Lovinger et al. ${ }^{14}$ observed a similar peak in $\varepsilon^{\prime}$ near the melting point for the copolymer with $19 \mathrm{~mol} \% \mathrm{TeFE}$ and ascribed it to the Curie transition.

In this paper, the Curie transition for VDF -TeFE copolymers with 17.8 to $30.7 \mathrm{~mol} \%$ TeFE was investigated in detail by thermal, 
dynamic mechanical and dielectric measurements. The effect of poling on the Curie transition was also examined by thermally stimulated current (TSC) and differential scanning calorimetry (DSC) measurements. The Curie points of these copolymers were compared with those of VDF-TrFE copolymers and discussed in viewpoint of some differences in crystal structures between copolymers of VDF with TeFE and TrFE.

\section{EXPERIMENTAL}

The samples used were the same VDFTeFE copolymers with 17.8, 25.1, and 30.7 $\mathrm{mol} \% \mathrm{TeFE}$ as those employed in the previous work. ${ }^{15,16}$ Powder samples were heat-pressed into films with thickness of 100 to $200 \mu \mathrm{m}$ at $523 \mathrm{~K}$. Two cooling conditions were used to obtain films with varied degrees of crystallinity: quenching specimen from $523 \mathrm{~K}$ into cold water and slow cooling from 523 to $320 \mathrm{~K}$ in air. The samples are listed in Table $I$ in which samples 1,3 , and 5 are obtained by slow cooling and samples 2 and 4 by quenching. The measurements of density were made by the floating method in mixtures of carbon tetrachloride and 1,1,2,2-tetrabromoethane. Densities of the samples are also given in Table I. For the samples with $30.7 \mathrm{~mol} \%$, no significant difference was found in density between quenched and slowly cooled samples. Dynamic mechanical measurements were carried out at a frequency of $3.5 \mathrm{~Hz}$ in a temperature range of 173 to $393 \mathrm{~K}$ using a Toyo Baldwin Model DDV-II-C Reovibron. X-Ray measurements were performed by a Rigaku Denki diffractometer Geigerflex 2012. Thermal properties were measured by a Rigaku Denki differential thermal analyzer Thermoflex TG-DTA and a Perkin-Elmer differential scanning calorimeter Model DSC-1B. Dielectric measurements were made on film specimens using a three terminal electrode by an Ando-Denki Model TR-1C transformer bridge at a frequency range of 1 to $100 \mathrm{kHz}$ from room temperature (RT)
Table I. Densities of VDF-TeFE copolymer samples

\begin{tabular}{|c|c|c|}
\hline \multirow{2}{*}{$\begin{array}{c}\text { Sample } \\
\text { No. }\end{array}$} & TeFE content & Density \\
\hline & $\mathrm{mol}^{\circ} \%$ & $10^{3} \mathrm{~kg} \mathrm{~m}^{-3}$ \\
\hline 1 & 17.8 & 1.865 \\
\hline 2 & 17.8 & 1.849 \\
\hline 3 & 25.1 & 1.940 \\
\hline 4 & 25.1 & 1.890 \\
\hline 5 & 30.7 & 1.896 \\
\hline
\end{tabular}

to $410 \mathrm{~K}$. Polarization current vs. electric field curves were obtained using a high-voltage triangular wave at $0.1 \mathrm{~Hz}$ by the method described in elsewhere. ${ }^{17}$ TSC measurements were performed at heating rate of $1.7 \mathrm{~K} \mathrm{~min}^{-1}$ by the method described previously. ${ }^{18}$ Poling was made on thin films about $10-50 \mu \mathrm{m}$ thick with evapolated aluminum electrode using a high-voltage triangular wave.

\section{RESULTS AND DISCUSSION}

\section{Unpoled Samples}

Figure 1 shows the $\mathrm{X}$-ray diffraction curves for slowly cooled samples 1, 3, and 5. These copolymers exhibit a single peak around $19.35-19.9^{\circ}$ in the diffraction angle $2 \theta$, taking a similar crystal structure to that of PVDF from I. ${ }^{1,2}$ Thus, this peak corresponds to the unresolved $(200,110)$ reflection. As is seen in Figure 1, the diffraction angle of this peak decreases with increase of TeFE content. The crystal lattice expands with increase of TeFE content. Diffraction patterns from 33 to $37^{\circ}$ in $2 \theta$ are shown in Figure 2. Sample 1 exhibited a peak at $35.1^{\circ}$ and sample 3 showed a diffused peak from 34 to $36^{\circ}$. Sample 5 also showed a dull diffused peak at $33.9^{\circ}$. In the PVDF form I crystal, $-\mathrm{CF}_{2}$ groups deflect statistically to the right and left in the zigzag plane to eliminate crowding of fluorine atoms along the molecular chain direction. The deflection angle and the $c$-axis repeat found for PVDF form I were $7^{\circ}$ and $2.56 \AA$, respectively. ${ }^{2}$ The peak at $35.1^{\circ}$ for sample 

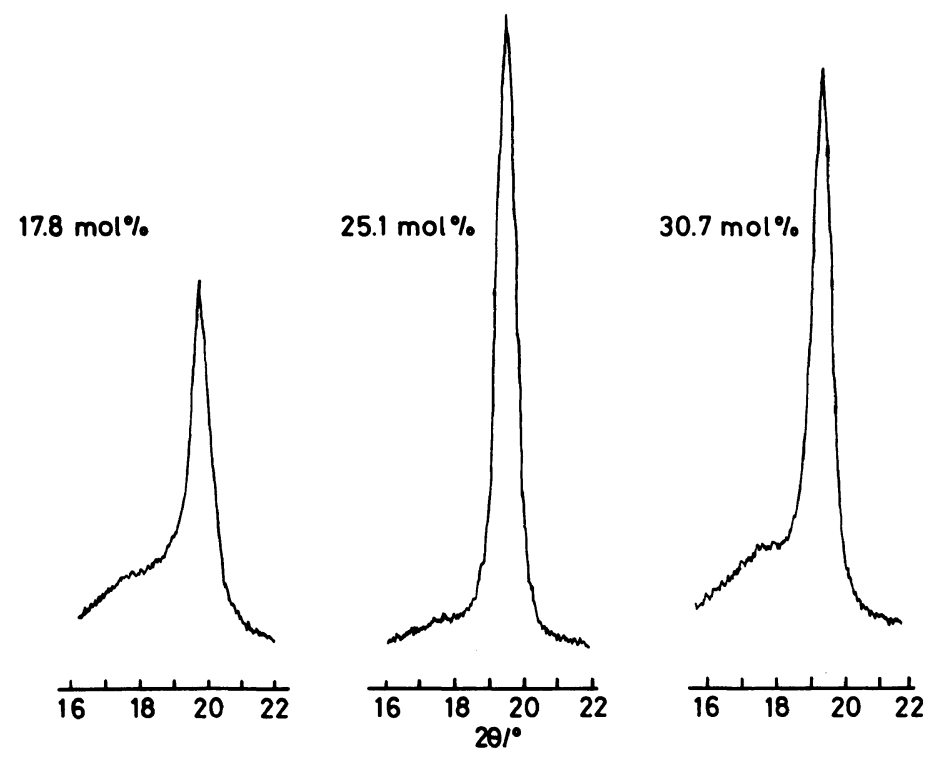

Figure 1. X-Ray diffraction patterns for slowly cooled samples 1,3 , and 5 from 16 to $22^{\circ}$ in diffraction angle $2 \theta$.

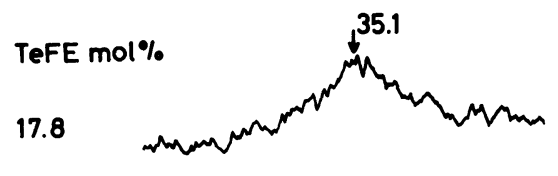

25.1

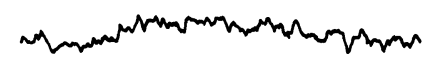

30.7

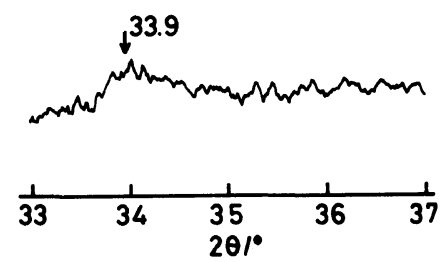

Figure 2. X-Ray diffraction patterns for the same samples as those in Figure 1.

1 corresponded to the (001) reflection of the all-trans crystal similar to that in PVDF form I. In polytetrafluoroethylene (PTeFE), the fluorine atoms must be more densely crowded than in PVDF because $-\mathrm{CH}_{2}$ groups are replaced by $-\mathrm{CF}_{2}$ groups in all-trans chains. Thus, conformation of PTeFE is a $13_{6}$ helix where the valence angle in $\mathrm{C}-\mathrm{C}-\mathrm{C}$ bond increases to $116^{\circ}$. The small peak at $33.9^{\circ}$ for sample 5 may be due to the $c$-axis repeat of the elongated all-trans chains which is caused by increase of valence angle in C$\mathrm{C}-\mathrm{C}$ bond found in PTeFE. This peak was so small that the $c$-axis repeat might be disordered for unpoled sample 5. In VDF-TrFE copolymers, the peak of (001) reflection remained still at $35 \mathrm{~mol} \%$ TrFE and the $(200$, 110) reflection was observed at a higher angle in $2 \theta$ than that for VDF-TeFE copolymers. ${ }^{3}$ Crowding of fluorine atom must be considerable more in VDF-TeFE copolymers than that in VDF-TrFE copolymers because of one more fluorine atom in each TeFE unit. It seems that the all-trans conformation deflects and elongates more for VDF-TeFE copolymers than those for VDF-TrFE copolymers. Thus the crystal lattice became large in $a$ - and $b$-axis directions and elongated in the $c$-axis repeat. Such change in the deflection angle and valence angle may be significant around TeFE units in sequences. Furthermore, it seems that the melt crystallized sample took the irregular conformation containing gauche and kink bonds as defects considered 


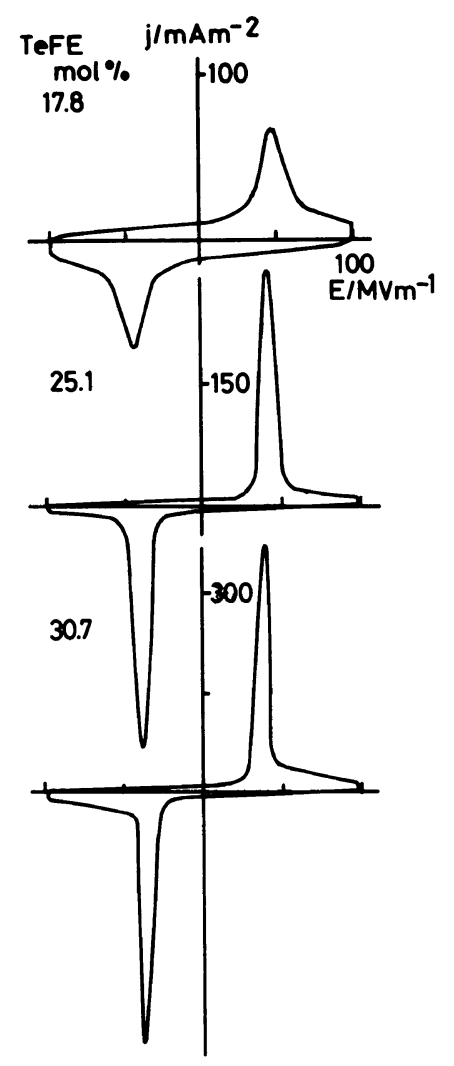

Figure 3. Polarization reversal current $v$ s. electric field curves for slowly cooled samples 1,3 , and 5 .

in the VDF-TrFE copolymer. ${ }^{19}$ These defects may take place in sequences near introduced TeFE units to eliminate crowding of the fluorine atom. These would give the disordered $c$-axis repeat. Density was highest at $25 \mathrm{~mol} \% \mathrm{TeFE}$ as seen in Table I, so the degree of crystallinity was highest at this content. The lower degree of crystallinity for sample 5 may be due to the disordered conformation induced by TeFE units. Ferroelectric properties were observed for VDFTeFE copolymers in this work. Figure 3 shows the polarization reversal currents $v s$. electric field curves for sample 1, 3, and 5 . A coercive field was $40-50 \mathrm{M} \mathrm{Vm}^{-1}$, being similar to that for the VDF-TrFE copolymers.

Dynamic tensile modulus $E^{\prime}$ and mecha-

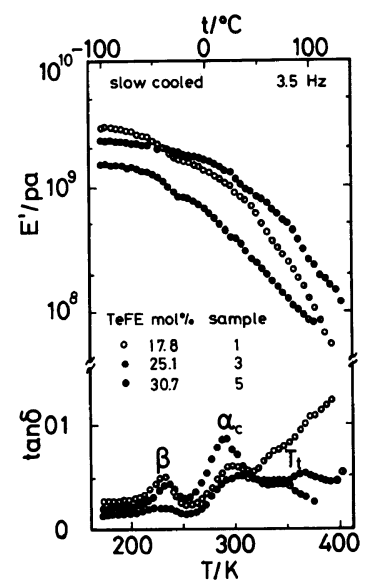

Figure 4. Temperature dependence of tensile modulus $E^{\prime}$ and mechanical loss tangent $\tan \delta$ at $3.5 \mathrm{~Hz}$ for slowly cooled samples 1,3 , and 5 .

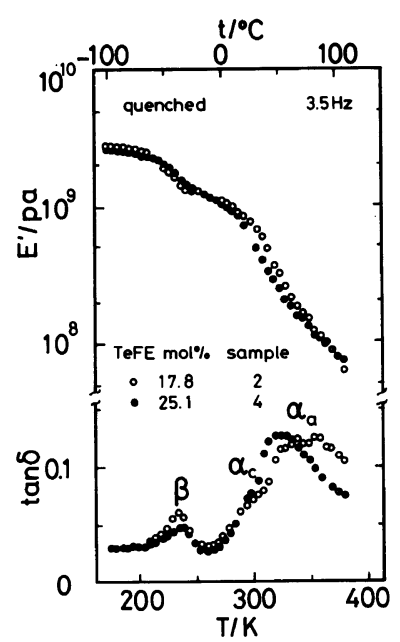

Figure 5. The same as Figure 4 for quenched samples 2 and 4.

nical loss tangent $\tan \delta$ are shown as a function of temperature for slowly cooled samples 1, 3, and 5 in Figure 4 and quenched samples 2 and 4 in Figure 5. Three relaxations, designated as $T_{\mathrm{t}}, \alpha_{\mathrm{c}}$ and $\beta$, were observed for slowly cooled samples. The $\alpha_{c}$ and $\beta$ relaxations were related to the molecular motions in the form I crystal and the micro-Brownian molecular motions in amorphous regions, respectively. Quenched samples exhibited the two peaks, designated as $\alpha_{\mathrm{a}}$ and $\beta$, and shoulder $\left(\alpha_{\mathrm{c}}\right)$ at the low 
temperature side on the $\alpha_{\mathrm{a}}$ relaxation. The $\alpha_{\mathrm{a}}$ relaxation in the quenched samples took place at higher temperature than the $\beta$ relaxation due to the micro-Brownian molecular motion. This relaxation would be due to the same relaxation mechanism as that in the $T_{\mathrm{g}}(U)$ relaxation, glass transition of chains constrained between crystallites, observed in PVDF. $^{21}$ The $T_{\mathrm{t}}$ relaxation was found for samples 3 and 5 with higher degree of crystallinity, being assigned to that of crystalline regions. As revealed in the previous paper, ${ }^{11}$ the mechanical relaxation was observed in the ferroelectric to paraelectric phase transition, i.e., the Curie transition, for the VDFTrFE copolymer and the tensile modulus decreased to 100 to $300 \mathrm{MPa}$ at the transition. The value of 100 to $300 \mathrm{MPa}$ may be related to the modulus in the paraelectric phase of crystal. Around the $T_{t}$ relaxation, the tensile modulus of VDF-TeFE copolymer decreased also to $100 \mathrm{MPa}$, so that the change of modulus was associated with the Curie transition. The $T_{\mathrm{t}}$ relaxation was not found for the copolymer with $17.8 \mathrm{~mol} \%$ TeFE.

DTA curves for slowly cooled samples 1, 3, and 5 are shown in Figure 6 where melting peaks are seen at 410 to $420 \mathrm{~K}$ and small endotherms are below the melting point. The temperatures of these small peaks were consistent with those of the $T_{\mathrm{t}}$ mechanical relaxation. Thus, the peaks are related to the Curie transition as observed in VDF-TrFE copolymers. ${ }^{10,12}$ The small endotherm observed near the melting point for sample $1 \cdot$ is also attributable to the Curie transition since variation of the Curie point with VDF content is linear and agrees with that for the VDFTeFE copolymer with $19 \mathrm{~mol} \%$ TeFE obtained by Lovinger et al., ${ }^{13,14}$ as shown in Figure 7. The most recent X-ray study ${ }^{20}$ for the Curie transition in these copolymers with 19 to $35 \mathrm{~mol} \%$ TeFE by Lovinger et al. gives good agreement with our data of the Curie temperature.

Dielectric constant and loss, $\varepsilon^{\prime}$ and $\varepsilon^{\prime \prime}$,

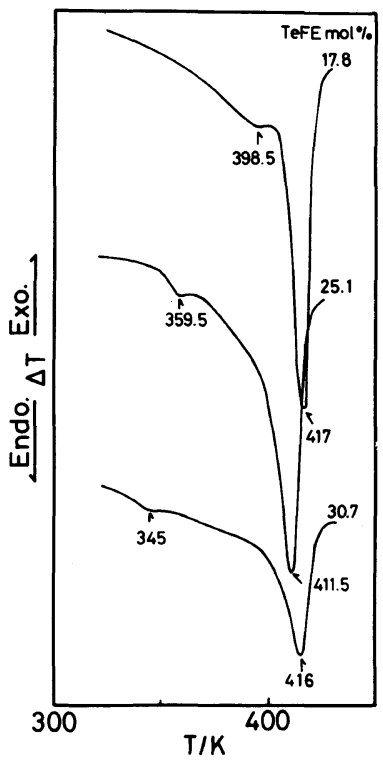

Figure 6. DTA curves for slowly cooled samples 1, 3, and 5 .

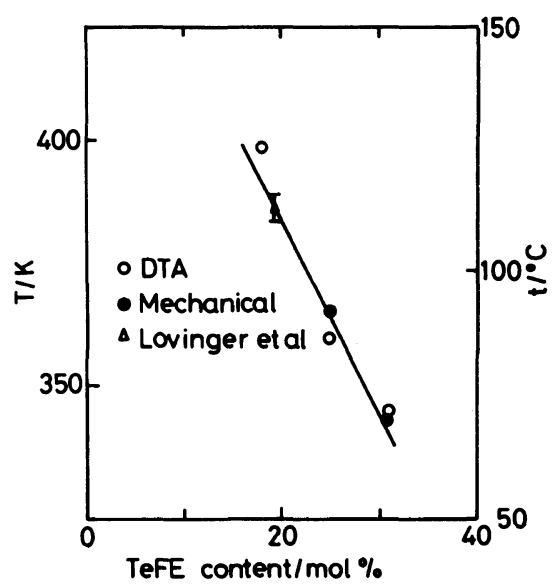

Figure 7. Variation of temperatures for the $T_{t}$ mechanical loss peak and DTA endotherm in Curie transition with TeFE content.

measured at $100 \mathrm{kHz}$ are shown as a function of temperature for the samples 1,3 , and 5 in Figure 8 . For sample 3 , the curve of $\varepsilon^{\prime \prime}$ shows a gradual increase from RT to $400 \mathrm{~K}$ and a shoulder around $370 \mathrm{~K}$. The curve of $\varepsilon^{\prime}$ exhibits a steep rise around $360 \mathrm{~K}$. This rise of $\varepsilon^{\prime}$ took place near the temperatures of the $T_{\mathrm{t}}$ mechanical relaxation and DTA endotherm, 


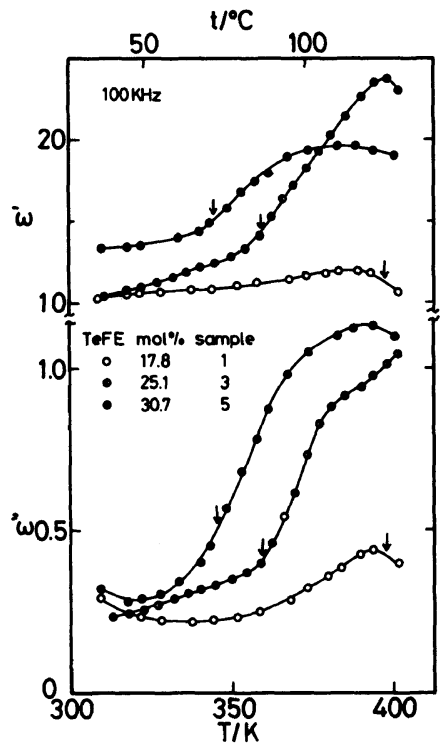

Figure 8. Temperature dependence dielectric constant $\varepsilon^{\prime}$ and loss $\varepsilon^{\prime \prime}$ at $100 \mathrm{kHz}$ for slowly cooled samples 1, 3, and 5 . Arrows show the Curie temperatures obtained by DTA measurements.

being attributable to the Curie transition. However, the value of $\varepsilon^{\prime}$ still increases beyond the Curie transition, disagreeing with the Curie Weiss law. In the previous work, $\varepsilon^{\prime}$ and $\varepsilon^{\prime \prime}$ increased with increasing temperature and decreasing frequency and were reduced by the electrodialysis which lowered the content of ionic impurity. ${ }^{16}$ Thus this dielectric behavior was attributed to the space charge polarization of the ionic impurity. ${ }^{16}$ The increase in $\varepsilon^{\prime}$ above the Curie transition in these copolymers seems to be related to the space charge polarization as follow. Most space charges will be trapped on the surface of polar crystals below the Curie transition while released above the transition. Such released charges may contribute to the increase in $\varepsilon^{\prime}$ above the Curie temperature. The mobility of these charges will increase as temperature increases and therefore the values of $\varepsilon^{\prime}$ also increase with increasing temperature. The other effect of the space charges on the dielectric behavior was considered as that when there is inhomogeneous space charge, the Curie point slightly

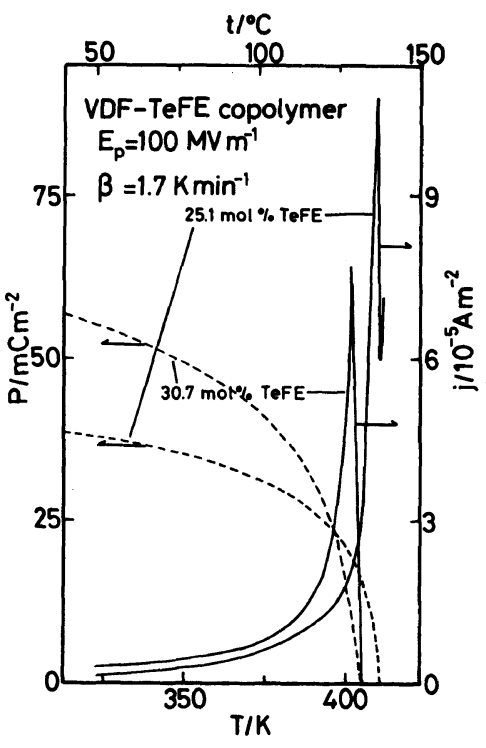

Figure 9. Temperature dependence of depolarization currents (solid line) and remnant polarization (dashed line) for poled samples 3 and 5 .

differs from part to part in a crystal and a peak of $\varepsilon^{\prime}$ will become round. ${ }^{22}$ The disordered crystal mentioned above may be also a reason for the difference of the Curie transition in each crystal. For sample 5 with $30.7 \mathrm{~mol} \%$ TeFE, a steep rise in $\varepsilon^{\prime}$ was observed at $345 \mathrm{~K}$ near the Curie point obtained by mechanical and DTA measurements in a similar manner to that of sample 3 . Therefore, it was considered that the steep increase of $\varepsilon^{\prime}$ of this copolymer is related also to the Curie transition. In case of the copolymer with $17.8 \mathrm{~mol} \%$ TeFE, a rather small peak was observed just below the melting point. Similar peaks were observed for sample 3 at $390 \mathrm{~K}$ and for sample 5 at $380-390 \mathrm{~K}$ so that it is difficult to judge whether these peaks are related to the Curie transition.

\section{Poled Sample}

Effects of poling on the Curie transition and crystal structure were examined for samples 3 and 5. TSC curves of these samples poled by triangular wave at $100 \mathrm{mHz}$ with the $\max$ imum electric field $E_{\mathrm{p}}$ of $100 \mathrm{MVm}^{-1}$ are 


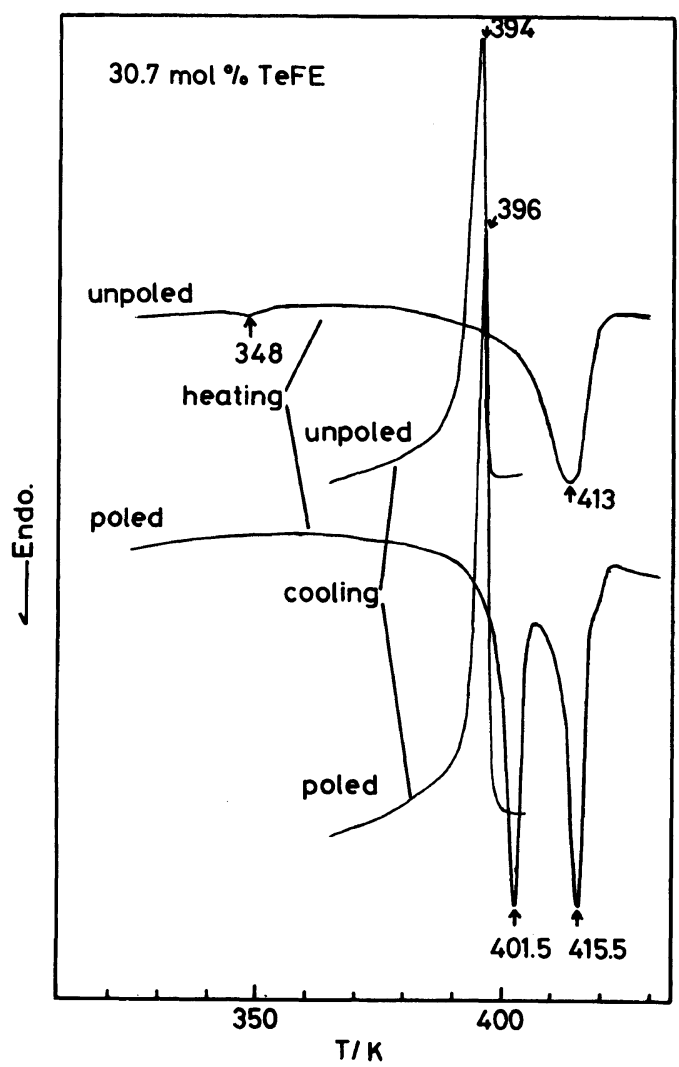

Figure 10. DSC curves for unpoled and poled sample 5 .

shown in Figure 9. Large TSC peaks were observed at 403 and $410 \mathrm{~K}$ for samples 5 and 3 , respectively. The polarizations estimated by integrating the TSC curves disappeared at 406 and $411 \mathrm{~K}$ for samples 3 and 5 , respectively, as shown in Figure 9. The DSC curves for sample 5 poled by the same condition as that in TSC measurements is shown together with that for the unpoled one in Figure 10. The small endotherm observed near $348 \mathrm{~K}$ in the unpoled sample disappeared and a considerably large peak appeared at $401.5 \mathrm{~K}$ for the poled sample. The temperature of this peak agreed with that at which the polarization disappeared. DSC curves for poled and unpoled sample 3 are shown in Figure 11. The poled sample showed a large peak at $409 \mathrm{~K}$ and an indistinct shoulder at $412 \mathrm{~K}$. It

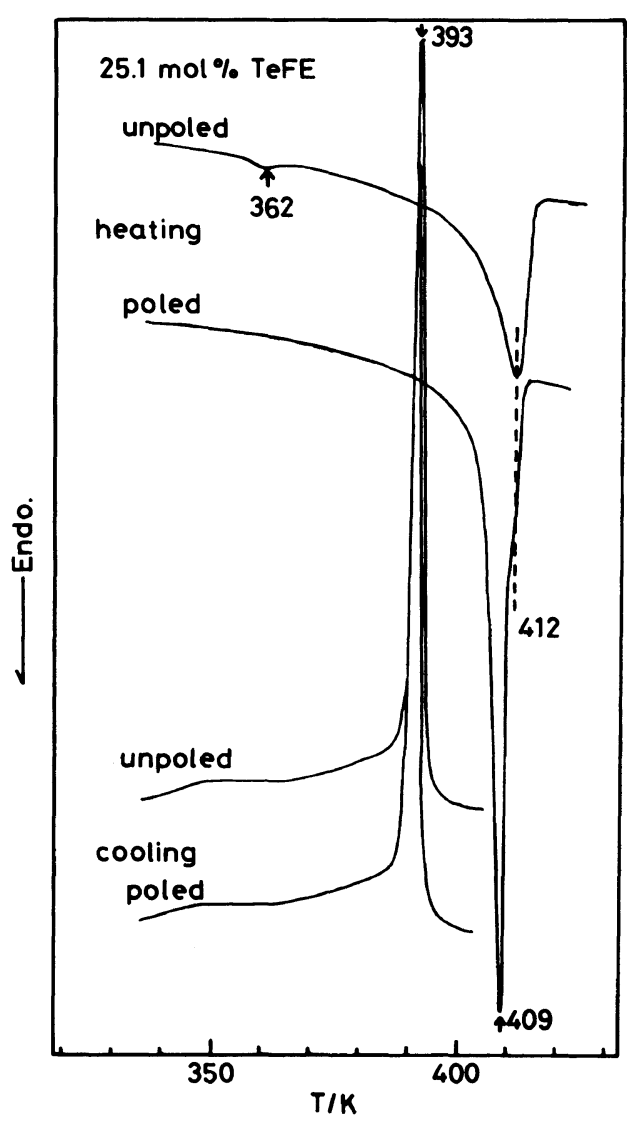

Figure 11. The same as Figure 10 for unpoled and poled sample 3 .

seems that the endothermic peak of fusion does not change considerably in size as shown for sample 5 in Figure 10. The indistinct shoulder for poled sample 3 took place at the same temperature as that of the melting peak for the unpoled sample, and thus, this shoulder is probably due to the fusion of crystals. The Curie points in these copolymers were elevated by $50 \mathrm{~K}$ in poling. Enthalpies of the Curie transition and fusion obtained were 0.4 and $19.5 \times 10^{3} \mathrm{~J} \mathrm{~kg}^{-1}$ for unpoled sample 5 and 16.8 and $19.0 \times 10^{3} \mathrm{~J}$ $\mathrm{kg}^{-1}$ for poled sample 5 , respectively. The increase of enthalpy was considerable for the Curie transition but not for fusion. Those of the Curie transition and the fusion for unpoled sample 3 were 0.8 and $46.0 \times 10^{3} \mathrm{~J} \mathrm{~kg}^{-1}$ 


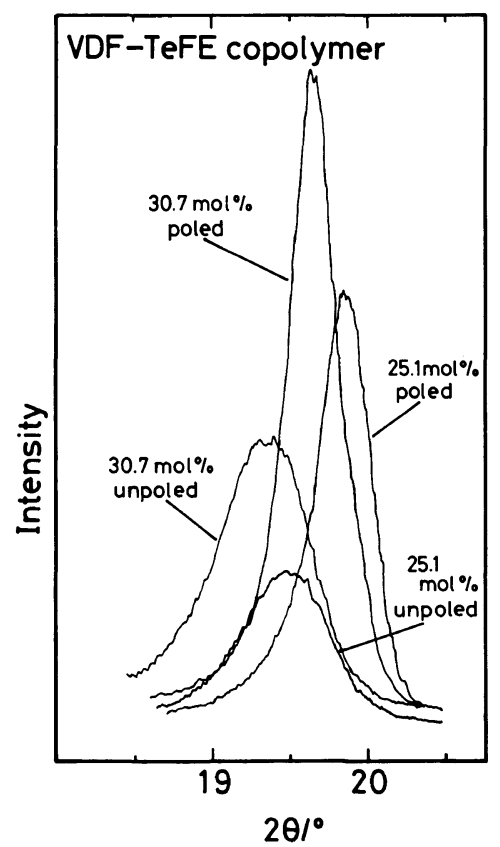

Figure 12. The same as Figure 1 for unpoled and poled samples 3 and 5 .

and total enthalpy of both transitions for poled sample 3 was $55.0 \times 10^{3} \mathrm{~J} \mathrm{~kg}^{-1}$. If the enthalpy of the fusion does not change for sample 3 in poling in the same way as for sample 5 , the enthalpy of the Curie transition is $9.0 \times 10^{3} \mathrm{~J} \mathrm{~kg}^{-1}$. The increases in the Curie temperature and enthalpy of the transition in VDF-TeFE copolymers by poling procedure were larger than those for the VDF-TrFE copolymers. In DSC curves for poled samples on cooling, the Curie transition behavior was not clear and the same profile as that for the unpoled sample was observed. This indicated that the ordered structure developed by poling is likely destroyed with melting of the crystal.

X-Ray diffraction patterns at 19 to $20^{\circ}$ in the diffraction angle $2 \theta$ are shown for poled and unpoled samples 3 and 5 in Figure 12. The main peak corresponding to the $(200$, 110) reflection is seen at 19.5 and $19.35^{\circ}$ for unpoled samples 3 and 5 , respectively. This peak shifted to higher angles, 19.85 and
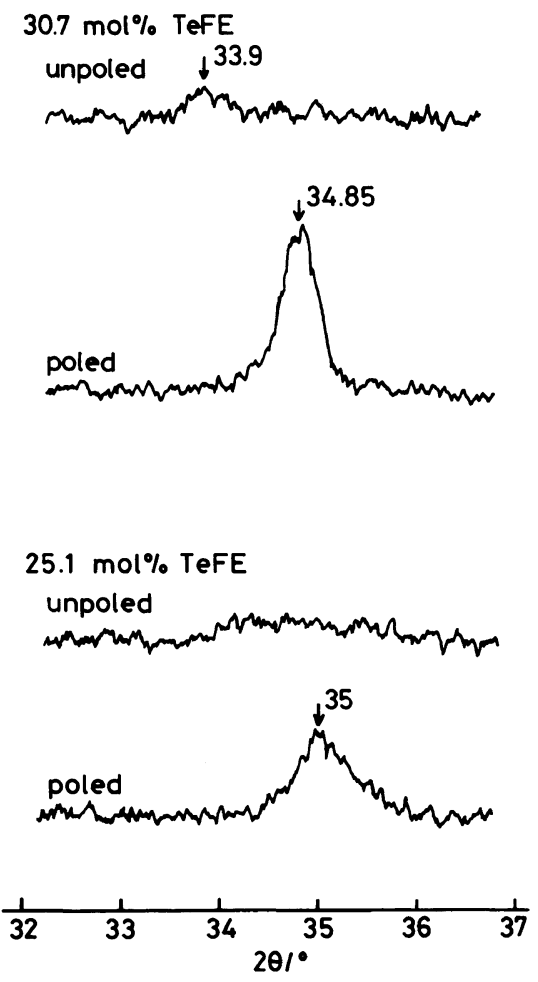

Figure 13. The same as Figure 2 for unpoled and poled samples 3 and 5 .

$19.65^{\circ}$ for poled samples 3 and 5 , respectively, by poling. The width of this peak at the half height decreased from 0.65 to $0.4^{\circ}$ for sample 5 and 0.60 to $0.4^{\circ}$ for sample 3 , by poling. Diffraction patterns at 32 to $37^{\circ}$ in $2 \theta$ are shown in Figure 13. A rather sharp peak was observed at 35 and $34.85^{\circ}$ for poled samples 3 and 5 , respectively, and the $c$-axis repeat was estimated as 2.56 and $2.57 \AA$. These facts indicate that the electric field converts the disordered chain conformation into a well ordered one, eliminates defects such as gauche and kink bonds and improves packing of chains in the crystals. The change of width at the half peak height was very large in the (001) peak, so that the size of the crystallites grew considerably in the $c$-axis direction. These changes in the crystals may give significant increase of the Curie points and the enthalpies of the transition for VDF-TeFE copolymers. 


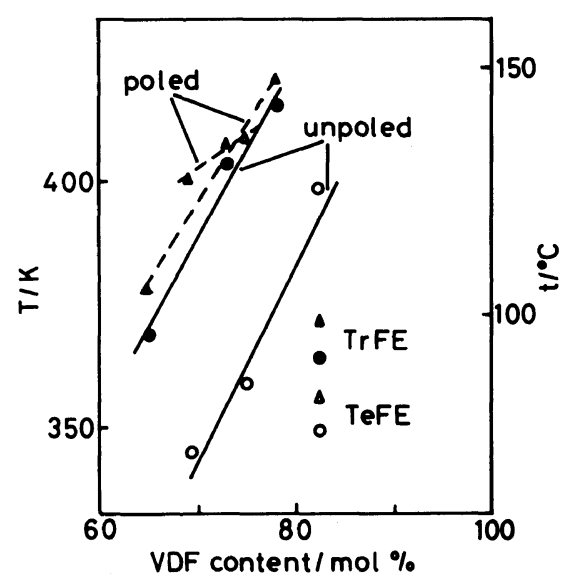

Figure 14. Variation of Curie point with VDF content for VDF-TeFE and VDF-TrFE copolymers. $\triangle$ and $O$, our data for VDF-TeFE copolymers; $\boldsymbol{\Delta}$ and $\boldsymbol{O}$, data for VDF-TrFE copolymers from ref 13.

In the VDF-TrFE copolymer with $35 \mathrm{~mol} \%$ TrFE, the $(200,110)$ peak shifted to higher angle in $2 \theta$ in a similar manner for VDF-TeFE copolymers by poling but the (001) peak clearly appeared even in the unpoled sample as shown by Lovinger et al. ${ }^{3}$ This copolymer may have the well ordered crystal and fewer defects even in the unpoled state so that change of conformation may be small. As a result, increase of the Curie point is smaller for VDFTrFE copolymers by poling than that for VDF-TeFE copolymers as shown in Figure 14. The increase in the degree of order and crystallinity by poling of the VDF-TeFE copolymer were also suggested by infrared and density measurements, respectively, by Latour. ${ }^{23}$ Densities increased also in this work from 1.940 to $1.962 \times 10^{3} \mathrm{~kg} \mathrm{~m}^{-3}$ and from 1.896 to $1.925 \times 10^{3} \mathrm{~kg} \mathrm{~m}^{-3}$ by poling samples 3 and 5, respectively. Crystal densities estimated from $\mathrm{X}$-ray data in this work were 2.055 and $2.063 \times 10^{3} \mathrm{~kg} \mathrm{~m}^{-3}$ for copolymers with 25.1 and $30.7 \mathrm{~mol}^{\circ} \%$ TeFE, respectively. Amorphous density was estimated by the method proposed by Van Krevelen. ${ }^{24}$ The degrees of crystallinity calculated were 65 and $46 \%$ for unpoled samples 3 and 5 and 72 and $56 \%$ for poled samples, respectively. It seems

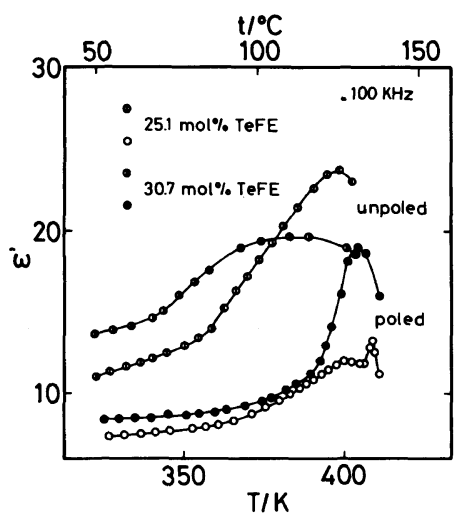

Figure 15. Temperature dependence of $\varepsilon^{\prime}$ for poled and unpoled samples 3 and 5 .

that this increase in degree of crystallinity for poled samples may be due to the ordered crystal structure.

Temperature dependence of $\varepsilon^{\prime}$ for poled samples 3 and 5 is shown in Figure 15 together with that for unpoled ones. In contrast to unpoled sample 5, a clear peak in $\varepsilon^{\prime}$ was observed for the poled one at the Curie temperature found in DSC measurements. Thus this peak may be connected with the Curie transition. For poled sample 3, a dull peak and small pointed peak are observed at 400 and $409 \mathrm{~K}$, respectively. The DSC measurements indicate that the peak at $409 \mathrm{~K}$ is related to the Curie transition; however, the peak size is very small. The melting takes place at $412 \mathrm{~K}$, disguising the Curie transition behavior of $\varepsilon^{\prime}$. The small pointed peak seems to be the early stage of the Curie transition. Below the transition, the values of $\varepsilon^{\prime}$ were reduced in both samples by poling. Dipoles should be forced to align cooperatively along the poling field direction so that molecular motions may be depressed by the local electric field. In addition, the space charge polarization seems to be suppressed also in the ferroelectric state, since the space charges may be trapped on the surface of polar crystal. 


\section{CONCLUSION}

The Curie transition was found for VDFTeFE copolymers with 17.8 to $30.7 \mathrm{~mol} \%$ TeFE. The transition temperature was lower for these copolymers than that for VDF-TrFE copolymers with the same VDF content. The peak due to the $(200,110)$ reflection was observed at lower angles in $2 \theta$ than that for VDF-TrFE copolymers and the $c$-axis repeat was not observed clearly. This fact indicated that chain conformation for VDF-TeFE copolymers was more disordered by introduction of TeFE units, so that the molecular chain was packed more loosely than that for VDFTrFE copolymers. The Curie temperature was elevated by about $50 \mathrm{~K}$ by poling. The $(200$, 110) reflection moved to higher angles in $2 \theta$ and the (001) reflection clearly appeared around $35^{\circ}$. Poling induces the well ordered molecular chain and improves packing of the molecular chain.

Acknowledgements. The author wishes to thank Professor Emeritus N. Koizumi of Kyoto University for his many valuable discussion and suggestions for this work. Thanks are also due to Dr. Y. Kubouchi and Mr. S. Koizumi of Daikin Kogyo Co., Ltd., Osaka for providing the samples, to Dr. N. Nakamura of Ritsumeikan University for making the DSC measurements and to Dr. T. Kobayashi of Kyoto University for use of the $\mathrm{X}$-ray diffractometer.

\section{REFERENCES}

1. J. B. Lando, H. G. Olf, and A. Peterlin, J. Polym.
Sci., A-1, 4, 941 (1966).

2. R. Hasegawa, Y. Takahashi, Y. Chatani, and H. Tadokoro, Polym. J., 3, 600 (1972).

3. A. J. Lovinger, T. Furukawa, G. T. Davis, and M. G. Broadhust, Polymer, 24, 1225 (1983).

4. K. Tashiro, K. Takano, M. Kobayashi, Y. Chatani, and H. Tadokoro, Polymer, 25, 195 (1984).

5. J. B. Lando and W. W. Dole, J. Macromol. Sci., Phys., B2, 205 (1968).

6. B. L. Farmer, A. J. Hopfinger, and J. B. Lando, J. Appl. Phys., 43, 4293 (1972).

7. J. C. Hicks, T. E. Jones, and J. C. Logan, J. Appl. Phys., 49, 6093 (1978).

8. S. Tasaka and S. Miyata, J. Appl. Phys., 57, 906 (1985).

9. T. Furukawa, M. Date, E. Fukada, Y. Tajitsu, and A. Chiba, Jpn. J. Appl. Phys., 19, L109 (1980).

10. T. Yagi, M. Tatemoto, and J. Sako, Polym. J., 12, 209 (1980).

11. Y. Murata and N. Koizumi, Polym. J., 17, 385 (1985).

12. N. Koizumi, N. Haikawa, and H. Habuka, Ferroelectrics, 57, 99 (1984).

13. A. J. Lovinger, Macromolecules, 16, 1529 (1983).

14. A. J. Lovinger, G. E. Johnson, H. E. Bair, and E. W. Anderson, J. Appl. Phys., 56, 2412 (1984).

15. Y. Murata and N. Koizumi, Polym. J., 17, 1071 (1985).

16. N. Koizumi, G. Hagino, and Y. Murata, Ferroelectrics, 32, 141 (1981)

17. N. Koizumi, Y. Murata, and H. Tsunashima, IEEE Trans., EI-21, 543 (1986).

18. Y. Oka and N. Koizumi, Polym. J., 14, 869 (1982).

19. H. Ohigashi and K. Koga, Jpn. J. Appl. Phys., 21, L455 (1982).

20. A. J. Lovinger, D. D. Davis, R. E. Cais, and J. M. Kometani, Macromolecules, 19, 1491 (1986).

21. R. F. Boyer, J. Polym. Sci., Polym. Symp., 50, 189 (1975).

22. T. Mitsui, I. Tatsuzaki, and E. Nakamura, "An Introduction to the Physics of Ferroelectrics," Gordon and Breach Science, New York, 1976.

23. M. Latour, Polymer, 18, 278 (1977).

24. D. W. Van Kreven and P. J. Hoftyzer, J. Appl. Polym. Sci., 13, 871 (1969). 